\title{
SECRECY WITHOUT ONE-WAY FUNCTIONS
}

\author{
DIMA GRIGORIEV AND VLADIMIR SHPILRAIN
}

\begin{abstract}
We show that some problems in information security can be solved without using one-way functions. The latter are usually regarded as a central concept of cryptography, but the very existence of one-way functions depends on difficult conjectures in complexity theory, most notably on the notorious " $P \neq N P$ " conjecture. This is why cryptographic primitives that do not employ one-way functions are often called "unconditionally secure".

In this paper, we suggest protocols for secure computation of the sum, product, and some other functions of two or more elements of an arbitrary constructible ring, without using any one-way functions. A new input that we offer here is that, in contrast with other proposals, we conceal "intermediate results" of a computation. For example, when we compute the sum of $k$ numbers, only the final result is known to the parties; partial sums are not known to anybody. Other applications of our method include voting/rating over insecure channels and a rather elegant and efficient solution of the "two millionaires problem".

Then, while it is fairly obvious that a secure (bit) commitment between two parties is impossible without a one-way function, we show that it is possible if the number of parties is at least 3 . We also show how our unconditionally secure (bit) commitment scheme for 3 parties can be used to arrange an unconditionally secure (bit) commitment between just two parties if they use a "dummy" (e.g., a computer) as the third party. We explain how our concept of a "dummy" is different from a wellknown concept of a "trusted third party". Based on a similar idea, we also offer an unconditionally secure $k$ - $n$ oblivious transfer protocol between two parties who use a "dummy".

We also suggest a protocol, without using a one-way function, for the so-called "mental poker", i.e., a fair card dealing (and playing) over distance.

Finally, we propose a secret sharing scheme where an advantage over Shamir's and other known secret sharing schemes is that nobody, including the dealer, ends up knowing the shares (of the secret) owned by any particular player.

It should be mentioned that computational cost of our protocols is negligible to the point that all of them can be executed without a computer.
\end{abstract}

\section{INTRODUCTION}

Secure multi-party computation is a problem that was originally suggested by Yao [18] in 1982. The concept usually refers to computational systems in which several parties wish to jointly compute some value based on individually held secret bits of

Research of the first author was partially supported by the Federal Agency of the Science and Innovations of Russia, State Contract No. 02.740.11.5192.

Research of the second author was partially supported by the NSF grants DMS-0914778 and CNS1117675 . 
information, but do not wish to reveal their secrets to anybody in the process. For example, two individuals who each possess some secret numbers, $x$ and $y$, respectively, may wish to jointly compute some function $f(x, y)$ without revealing any information about $x$ or $y$ other than what can be reasonably deduced by knowing the actual value of $f(x, y)$.

Secure computation was formally introduced by Yao as secure two-party computation. His "two millionaires problem" (cf. our Section 3) and its solution gave way to a generalization to multi-party protocols, see e.g. [4], [7]. Secure multi-party computation provides solutions to various real-life problems such as distributed voting, private bidding and auctions, sharing of signature or decryption functions, private information retrieval, etc.

In this paper, we offer protocols for secure computation of the sum and product of three or more elements of an arbitrary constructible ring without using encryption or any one-way functions whatsoever. We require in our scheme that there are $k$ secure channels for communication between the $k \geq 3$ parties, arranged in a circuit. We also show that less than $k$ secure channels is not enough.

Unconditionally secure multiparty computation was previously considered in [4] and elsewhere (since the present paper is not a survey, we do not give a comprehensive bibliography on the subject here, but only mention what is most relevant to our paper). A new input that we offer here is that, in contrast with [4] and other proposals, we conceal "intermediate results" of a computation. For example, when we compute a sum of $k$ numbers $n_{i}$, only the final result $\sum_{i=1}^{k} n_{i}$ is known to the parties; partial sums are not known to anybody. This is not the case in [4] where each partial sum $\sum_{i=1}^{s} n_{i}$ is known to at least some of the parties. This difference is important because, by the "pigeonhole principle", at least one of the parties may accumulate sufficiently many expressions in $n_{i}$ to be able to recover at least some of the $n_{i}$ other than his own.

Here we show how our method works for computing the sum (Section 2) and the product (Section 4) of private numbers. We ask what other functions can be securely computed without revealing intermediate results.

Other applications of our method include voting/rating over insecure channels (Section 2.3) and a rather elegant solution of the "two millionaires problem" (Section 3).

We also address another cryptographic primitive, known as (bit) commitment. In cryptography, a commitment scheme allows one to commit to a value while keeping it hidden, with the ability to reveal the committed value later. Commitments are used to bind a party to a value so that they cannot adapt to other messages in order to gain some kind of inappropriate advantage. They are important to a variety of cryptographic protocols including secure coin flipping, zero-knowledge proofs, and secure multi-party computation. See [8] or [13] for a general overview.

It is known [12] that a secure (bit) commitment between two parties is impossible without some kind of encryption, i.e., without a one-way function. However, if the number of parties is at least 3, this becomes possible, as long as parties do not form coalitions to trick other party (or parties). It has to be pointed out though that formal definitions of commitment schemes vary strongly in notation and in flavor, so we have 
to be specific about our model. We give more formal details in Section 6, while here we just say, informally, that what we achieve is the following: if the committed values are just bits, then after the commitment stage of our scheme is completed, none of the parties can guess any other party's bit with probability greater than $\frac{1}{2}$. We require in our scheme that there are $k$ secure channels for communication between the parties, arranged in a circuit. We also show that less than $k$ secure channels is not enough.

Then, in Section 7, we show how our unconditionally secure (bit) commitment scheme for 3 parties can be used to arrange an unconditionally secure (bit) commitment between just two parties if they use a "dummy" (e.g., a computer) as the third party. We explain how our concept of a "dummy" is different from a well-known concept of a "trusted third party" and also from Rivest's idea of a "trusted initializer" [15]. In particular, a difference important for real-life applications is that our "dummy" is unaware of the committed values. Also, our "dummy" is passive, i.e., he does not privately transmit information to "real" participants and he does not generate randomness.

Based on a similar idea, we also offer, in Section 8, an unconditionally secure $k$ - $n$ oblivious transfer protocol between two parties who use a "dummy".

In Section 9, we consider a related cryptographic primitive known as "mental poker", i.e., a fair card dealing (and playing) over distance. Several protocols for doing this, most of them using encryption, have been suggested, the first by Shamir, Rivest, and Adleman [17], and subsequent proposals include [5] and [9]. As with the bit commitment, a fair card dealing between just two players over distance is impossible without a one-way function since commitment is part of any meaningful card dealing scenario. However, it turns out to be possible if the number of players is $k \geq 3$. What we require though is that there are $k$ secure channels for communication between players, arranged in a circuit. We also show that our protocol can, in fact, be adapted to deal cards to just 2 players. Namely, if we have 2 players, they can use a "dummy" player (e.g. a computer), deal cards to 3 players, and then just ignore the "dummy"'s cards, i.e., "put his cards back in the deck". An assumption on the "dummy" player is that he cannot generate any randomness, so randomness has to be supplied to him by the two "real" players. Another assumption is that there are secure channels for communication between either "real" player and the "dummy". We believe that this model is adequate for 2 players who want to play online but do not trust the server. "Not trusting" the server exactly means not trusting with generating randomness. Other, deterministic, operations can be verified at the end of the game; we give more details in Section 9.2.

We note that the only known (to us) proposal for dealing cards to $k \geq 3$ players over distance without using one-way functions was published in [1], but their protocol lacks the simplicity, efficiency, and some of the functionalities of our proposal; this is discussed in more detail in our Section 10. Here we just mention that computational cost of our protocols is negligible to the point that they can be easily executed without a computer.

Finally, in Section 11, we propose a secret sharing scheme where an advantage over Shamir's [16] and other known secret sharing schemes is that nobody, including the dealer, ends up knowing the shares (of the secret) owned by any particular players. The disadvantage though is that our scheme is a $(k, k)$-threshold scheme only. 


\section{Secure computation of a SUM}

In this section, our scenario is as follows. There are $k$ parties $P_{1}, \ldots, P_{k}$; each $P_{i}$ has a private element $n_{i}$ of a fixed constructible ring $R$. The goal is to compute the sum of all $n_{i}$ without revealing any of the $n_{i}$ to any party $P_{j}, j \neq i$.

One obvious way to achieve this is well studied in the literature (see e.g. [8, 9, 11]): encrypt each $n_{i}$ as $E\left(n_{i}\right)$, send all $E\left(n_{i}\right)$ to some designated $P_{i}$ (who does not have a decryption key), have $P_{i}$ compute $S=\sum_{i} E\left(n_{i}\right)$ and send the result to the participants for decryption. Assuming that the encryption function $E$ is homomorphic, i.e., that $\sum_{i} E\left(n_{i}\right)=E\left(\sum_{i} n_{i}\right)$, each party $P_{i}$ can recover $\sum_{i} n_{i}$ upon decrypting $S$.

This scheme requires not just a one-way function, but a one-way function with a trapdoor since both encryption and decryption are necessary to obtain the result.

What we suggest in this section is a protocol that does not require any one-way function, but involves secure communication between some of the $P_{i}$. So, our assumption here is that there are $k$ secure channels of communication between the $k$ parties $P_{i}$, arranged in a circuit. Our result is computing the sum of private elements $n_{i}$ without revealing any individual $n_{i}$ to any $P_{j}, j \neq i$. Clearly, this is only possible if the number of participants $P_{i}$ is greater than 2. As for the number of secure channels between $P_{i}$, we will show that it cannot be less than $k$, by the number of parties.

\subsection{The protocol (computing the sum).}

(1) $P_{1}$ initiates the process by sending $n_{1}+n_{01}$ to $P_{2}$, where $n_{01}$ is a random element ("noise").

(2) Each $P_{i}, 2 \leq i \leq k-1$, does the following. Upon receiving an element $m$ from $P_{i-1}$, he adds his $n_{i}+n_{0 i}$ to $m$ (where $n_{0 i}$ is a random element) and sends the result to $P_{i+1}$.

(3) $P_{k}$ adds $n_{k}+n_{0 k}$ to whatever he has received from $P_{k-1}$ and sends the result to $P_{1}$.

(4) $P_{1}$ subtracts $n_{01}$ from what he got from $P_{k}$; the result now is the sum $S=$ $\sum_{1 \leq i \leq k} n_{i}+\sum_{2 \leq i \leq k} n_{0 i}$. Then $P_{1}$ publishes $S$.

(5) Now all participants $P_{i}$, except $P_{1}$, broadcast their $n_{0 i}$, possibly over insecure channels, and compute $\sum_{2 \leq i \leq k} n_{0 i}$. Then they subtract the result from $S$ to finally get $\sum_{1 \leq i \leq k} n_{i}$.

Thus, in this protocol we have used $k$ (by the number of the parties $P_{i}$ ) secure channels of communication between the parties. If we visualize the arrangement as a graph with $k$ vertices corresponding to the parties $P_{i}$ and $k$ edges corresponding to secure channels, then this graph will be a $k$-cycle. Other arrangements are possible, too; in particular, a union of disjoint cycles of length $\geq 3$ would do. (In that case, the graph will still have $k$ edges.) Two natural questions that one might now ask are: (1) is any arrangement with less than $k$ secure channels possible? (2) with $k$ secure channels, would this scheme work with any arrangement other than a union of disjoint cycles of length $\geq 3$ ? The answer to both questions is "no". Indeed, if there is a vertex (corresponding to $P_{1}$, say) of degree 0 , then any information sent out by $P_{1}$ will be available to everybody, so other participants will know $n_{1}$ unless $P_{1}$ uses a one-way 
function to conceal it. If there is a vertex (again, corresponding to $P_{1}$ ) of degree 1 , this would mean that $P_{1}$ has a secure channel of communication with just one other participant, say $P_{2}$. Then any information sent out by $P_{1}$ will be available at least to $P_{2}$, so $P_{2}$ will know $n_{1}$ unless $P_{1}$ uses a one-way function to conceal it. Thus, every vertex in the graph should have degree at least 2 , which implies that every vertex is included in a cycle. This immediately implies that the total number of edges is at least $k$. If now a graph $\Gamma$ has $k$ vertices and $k$ edges, and every vertex of $\Gamma$ is included in a cycle, then every vertex has degree exactly 2 since by the "handshaking lemma" the sum of the degrees of all vertices in any graph equals twice the number of edges. It follows that our graph is a union of disjoint cycles.

2.2. Effect of coalitions. Suppose now we have $k \geq 3$ parties with $k$ secure channels of communication arranged in a circuit, and suppose 2 of the parties secretly form a coalition. Our assumption here is that, because of the circular arrangement of secure channels, a secret coalition is only possible between parties $P_{i}$ and $P_{i+1}$ for some $i$, where the indices are considered modulo $k$; otherwise, attempts to form a coalition (over insecure channels) will be detected. If two parties $P_{i}$ and $P_{i+1}$ exchanged information, they would, of course, know each other's elements $n_{i}$, but other than that, they would not get any advantage if $k \geq 4$. Indeed, we can just "glue these two parties together", i.e., consider them as one party, and then the protocol is essentially reduced to that with $k-1 \geq 3$ parties. On the other hand, if $k=3$, then, of course, two parties together have all the information about the third party's element.

For an arbitrary $k \geq 4$, if $n<k$ parties want to form a (secret) coalition to get information about some other party's element, all these $n$ parties have to be connected by secure channels, which means there is a $j$ such that these $n$ parties are $P_{j}, P_{j+1}, \ldots, P_{j+n-1}$, where indices are considered modulo $k$. It is not hard to see then that only a coalition of $k-1$ parties $P_{1}, \ldots, P_{i-1}, P_{i+1}, \ldots, P_{k}$ can suffice to get information about the $P_{i}$ 's element.

2.3. Ramification: voting/rating over insecure channels. In this section, our scenario is as follows. There are $k$ parties $P_{1}, \ldots, P_{k}$; each $P_{i}$ has a private integer $n_{i}$. There is also a computing entity $B$ (for Boss) who shall compute the sum of all $n_{i}$. The goal is to let $B$ compute the sum of all $n_{i}$ without revealing any of the $n_{i}$ to him or to any party $P_{j}, j \neq i$.

The following example from real life is a motivation for this scenario.

Example 1. Suppose members of the board in a company have to vote for a project by submitting their numeric scores (say, from 1 to 10) to the president of the company. The project gets a green light if the total score is above some threshold value T. Members of the board can discuss the project between themselves and exchange information privately, but none of them wants his/her score to be known to either the president or any other member of the board.

In the protocol below, we are again assuming that there are $k$ channels of communication between the parties, arranged in a circuit: $P_{1} \rightarrow P_{2} \rightarrow \ldots \rightarrow P_{k} \rightarrow P_{1}$. On the 
other hand, communication channels between B and any of the parties are not assumed to be secure.

\subsection{The protocol (rating over insecure channels).}

(1) $P_{1}$ initiates the process by sending $n_{1}+n_{01}$ to $P_{2}$, where $n_{01}$ is a random number.

(2) Each $P_{i}, 2 \leq i \leq k-1$, does the following. Upon receiving a number $m$ from $P_{i-1}$, he adds his $n_{i}+n_{0 i}$ to $m$ (where $n_{0 i}$ is a random number) and sends the result to $P_{i+1}$.

(3) $P_{k}$ adds $n_{k}+n_{0 k}$ to whatever he has received from $P_{k-1}$ and sends the result to B.

(4) $P_{k}$ now starts the process of collecting the "adjustment" in the opposite direction. To that effect, he sends his $n_{0 k}$ to $P_{k-1}$.

(5) $P_{k-1}$ adds $n_{0(k-1)}$ and sends the result to $P_{k-2}$

(6) The process ends when $P_{1}$ gets a number from $P_{2}$, adds his $n_{01}$, and sends the result to $\mathrm{B}$. This result is the sum of all $n_{0 i}$.

(7) B subtracts what he got from $P_{1}$ from what he got from $P_{k}$; the result now is the sum of all $n_{i}, 1 \leq i \leq k$.

\section{Applichtion: the "TWo millionaires problem"}

The protocol from Section 2, with some adjustments, can be used to provide an elegant and efficient solution to the "two millionaires problem" introduced in [18]: there are two numbers, $n_{1}$ and $n_{2}$, and the goal is to solve the inequality $n_{1} \geq n_{2}$ ? without revealing the actual values of $n_{1}$ or $n_{2}$.

To that effect, we use a "dummy" as the third party. Our concept of a "dummy" is quite different from a well-known concept of a "trusted third party"; importantly, our "dummy" is not supposed to generate any randomness; he just does what he is told to. Basically, the only difference between our "dummy" and a usual calculator is that there are secure channels of communication between the "dummy" and either "real" party. One possible real-life interpretation of such a "dummy" would be an online calculator that can combine inputs from different users. Also note that in our scheme below the "dummy" is unaware of the committed values of $n_{1}$ or $n_{2}$, which is useful in case the two "real" parties do not want their private numbers to ever be revealed. This suggests yet another real-life interpretation of a "dummy", where he is a mediator between two parties negotiating a settlement.

Thus, let A (Alice) and B (Bob) be two "real" parties, and D (Dummy) the "dummy". Suppose A's number is $n_{1}$, and B's number is $n_{2}$.

\subsection{The protocol (comparing two numbers).}

(1) A splits her number $n_{1}$ as a difference $n_{1}=n_{1}^{+}-n_{1}^{-}$. She then sends $n_{1}^{-}$to B.

(2) B splits his number $n_{2}$ as a difference $n_{2}=n_{2}^{+}-n_{2}^{-}$. He then sends $n_{2}^{-}$to A.

(3) A sends $n_{1}^{+}+n_{2}^{-}$to D.

(4) B sends $n_{2}^{+}+n_{1}^{-}$to D. 
(5) D subtracts $\left(n_{2}^{+}+n_{1}^{-}\right)$from $\left(n_{1}^{+}+n_{2}^{-}\right)$to get $n_{1}-n_{2}$, and announces whether this result is positive or negative.

Remark 1. Perhaps a point of some dissatisfaction in this protocol could be the fact that the "dummy" ends up knowing the actual difference $n_{1}-n_{2}$, so if there is a leak of this information to either party, this party would recover the other's private number $n_{i}$. This can be avoided if $n_{1}$ and $n_{2}$ are represented in the binary form and compared one bit at a time, going left to right, until the difference between bits becomes nonzero. However, this method, too, has a disadvantage: the very moment the "dummy" pronounces the difference between bits nonzero would give an estimate of the difference $n_{1}-n_{2}$ to the real parties, not just to the "dummy".

We note that the original solution of the "two millionaires problem" given in [18], although lacks the elegance of our scheme, does not involve a third party, whereas our solution does. On the other hand, the solution in [18] uses encryption, whereas our solution does not, which makes it by far more efficient.

\section{Secure computation of a PRoduct}

In this section, we show how to use the same general ideas from Section 2 to securely compute a product. Again, there are $k$ parties $P_{1}, \ldots, P_{k}$; each $P_{i}$ has a private (nonzero) element $n_{i}$ of a fixed constructible ring $R$. The goal is to compute the product of all $n_{i}$ without revealing any of the $n_{i}$ to any party $P_{j}, j \neq i$. Requirements on the ring $R$ are going to be somewhat more stringent here than they were in Section 2 . Namely, we require that $R$ does not have zero divisors and, if an element $r$ of $R$ is a product $a \cdot x$ with a known $a$ and an unknown $x$, then $x$ can be efficiently recovered from $a$ and $r$. Examples of rings with these properties include the ring of integers and any constructible field.

\subsection{The protocol (computing the product).}

(1) $P_{1}$ initiates the process by sending $n_{1} \cdot n_{01}$ to $P_{2}$, where $n_{01}$ is a random nonzero element ("noise").

(2) Each $P_{i}, 2 \leq i \leq k-1$, does the following. Upon receiving an element $m$ from $P_{i-1}$, he multiplies $m$ by $n_{i} \cdot n_{0 i}$ (where $n_{0 i}$ is a random element) and sends the result to $P_{i+1}$.

(3) $P_{k}$ multiplies by $n_{k} \cdot n_{0 k}$ whatever he has received from $P_{k-1}$ and sends the result to $P_{1}$. This result is the product $P=\Pi_{1 \leq i \leq k} n_{i} \cdot \Pi_{2 \leq i \leq k} n_{0 i}$.

(4) $P_{1}$ divides what he got from $P_{k}$ by his $n_{01}$; the result now is the product $P=\Pi_{1 \leq i \leq k} n_{i} \cdot \Pi_{2 \leq i \leq k} n_{0 i}$. Then $P_{1}$ publishes $P$.

(5) Now all participants $P_{i}$, except $P_{1}$, broadcast their $n_{0 i}$, possibly over insecure channels, and compute $\Pi_{2 \leq i \leq k} n_{0 i}$. Then they divide $P$ by the result to finally get $\Pi_{1 \leq i \leq k} n_{i}$. 


\section{SeCURe COMputation OF Symmetric FunCtions}

In this section, we show how our method can be easily generalized to allow secure computation of any expression of the form $\sum_{i=1}^{k} n_{i}^{r}$, where $n_{i}$ are parties' private numbers, $k$ is the number of parties, and $r \geq 1$ an arbitrary integer. We simplify our method here by removing the "noise", to make the exposition more transparent.

\subsection{The protocol (computing the sum of powers).}

(1) $P_{1}$ initiates the process by sending a random element $n_{0}$ to $P_{2}$.

(2) Each $P_{i}, 2 \leq i \leq k-1$, does the following. Upon receiving an element $m$ from $P_{i-1}$, he adds his $n_{i}^{r}$ to $m$ and sends the result to $P_{i+1}$.

(3) $P_{k}$ adds his $n_{k}^{r}$ to whatever he has received from $P_{k-1}$ and sends the result to $P_{1}$.

(4) $P_{1}$ subtracts $\left(n_{0}-n_{1}^{r}\right)$ from what he got from $P_{k}$; the result now is the sum of all $n_{i}^{r}, 1 \leq i \leq k$.

Now that the parties can securely compute the sum of any powers of their $n_{i}$, they can also compute any symmetric function of $n_{i}$. However, in the course of computing a symmetric function from sums of different powers of $n_{i}$, at least some of the parties will possess several different polynomials in $n_{i}$, so chances are that at least some of the parties will be able to recover at least some of the $n_{i}$. On the other hand, because of the symmetry of all expressions involved, there is no way to tell which $n_{i}$ belongs to which party.

\subsection{Open problem. Now it is natural to ask:}

Problem 1. What other functions (other than the sum and the product) can be securely computed without revealing intermediate results to any party?

To be more precise, we note that one intermediate result is inevitably revealed to the party who finishes computation, but this cannot be avoided in any scenario. For example, after the parties have computed the sum of their private numbers, each party also knows the sum of all numbers except his own. What we want is that no other intermediate results are ever revealed.

To give some insight into this problem, we consider a couple of examples of computing simple functions different from the sum and the product of the parties' private numbers.

Example 2. We show how to compute the function $f\left(n_{1}, n_{2}, n_{3}\right)=n_{1} n_{2}+n_{2} n_{3}$ in the spirit of the present paper, without revealing (or even computing) any intermediate results, i.e., without computing $n_{1} n_{2}$ or $n_{2} n_{3}$.

(1) $P_{2}$ initiates the process by sending a random element $n_{0}$ to $P_{3}$.

(2) $P_{3}$ adds his $n_{3}$ to $n_{0}$ and sends $n_{3}+n_{0}$ to $P_{1}$.

(3) $P_{1}$ adds his $n_{1}$ to $n_{0}+n_{3}$ and sends the result to $P_{2}$.

(4) $P_{2}$ subtracts $n_{0}$ from $n_{0}+n_{3}+n_{1}$ and multiplies the result by $n_{2}$. This is now $n_{1} n_{2}+n_{2} n_{3}$. 
Example 3. The point of this example is to show that functions that can be computed by our method do not have to be homogeneous (in case the reader got this impression based on the previous examples).

The function that we compute here is $f\left(n_{1}, n_{2}, n_{3}\right)=n_{1} n_{2}+g\left(n_{3}\right)$, where $g$ is any computable function.

(1) $P_{1}$ initiates the process by sending a random element $a_{0}$ to $P_{2}$.

(2) $P_{2}$ multiplies $a_{0}$ by his $n_{2}$ and sends the result to $P_{3}$.

(3) $P_{3}$ multiplies $a_{0} n_{2}$ by a random element $c_{0}$ and sends the result to $P_{1}$.

(4) $P_{1}$ multiplies $a_{0} n_{2} c_{0}$ by his $n_{1}$, divides by $a_{0}$, and sends the result, which is $n_{1} n_{2} c_{0}$, back to $P_{3}$.

(5) $P_{3}$ divides $n_{1} n_{2} c_{0}$ by $c_{0}$ and adds $g\left(n_{3}\right)$, to end up with $n_{1} n_{2}+g\left(n_{3}\right)$.

Note that in this example, the parties used more than just one loop of transmissions in the course of computation. Also, information here was sent "in both directions" in the circuit.

Remark 2. Another collection of examples of multiparty computation without revealing intermediate results can be obtained as follows. Suppose, without loss of generality, that some function $f\left(n_{1}, \ldots, n_{k}\right)$ can be computed by our method in such a way that the last step in the computation is performed by the party $P_{1}$, i.e., $P_{1}$ is the one who ends up with $f\left(n_{1}, \ldots, n_{k}\right)$ while no party knows any intermediate result $g\left(n_{1}, \ldots, n_{k}\right)$ of this computation. Then, obviously, $P_{1}$ can produce any function of the form $F\left(n_{1}, f\left(n_{1}, \ldots, n_{k}\right)\right)$ (for a computable function $F$ ) as well. Examples include $n_{1}^{r}+n_{1} n_{2} \cdots n_{k}$ for any $r \geq 0$; $n_{1}^{r}+\left(n_{1} n_{2}+n_{3}\right)^{s}$ for any $r, s \geq 0$, etc., etc.

\section{6. (BIT) COMMITMENT}

While it is fairly obvious that a secure (bit) commitment between two parties is impossible without a one-way function, we show here that it is possible if the number of parties is at least 3. Generalizing the standard concept (see e.g. [8]) of a two-party (bit) commitment scheme, we define an $n$-party (bit) commitment scheme to be a twophase protocol through which each of the $n$ parties can commit himself to a value such that the following two requirements are satisfied:

(1) Secrecy: at the end of the commitment phase, none of the $n$ parties gains any information about any other party's committed value.

(2) Unambiguity: suppose that the commitment phase is successfully completed. Then, if later the parties perform the decommitment phase (sometimes called the reveal phase), each party's committed value can be recovered (collectively by other parties) without ambiguity.

To make our ideas more transparent, we start with the simplest case where there are just 3 parties: $P_{1}, P_{2}$, and $P_{3}$, and no two of them form a coalition against the third one. Suppose they want to commit to integers $n_{1}, n_{2}$, and $n_{3}$ (modulo some $m \geq 2$ ), respectively. More precisely, the scenario is as follows. During the commitment phase, the parties exchange various pieces of information about their integers $n_{i}$. After that, 
the parties "decommit", or reveal, their integers and prove to each other that the integers $n_{i}$ that they revealed are the same that they committed to.

All computations below are performed modulo a fixed integer $m \geq 2$.

(1) Each participant $P_{i}$ randomly splits his integer $n_{i}$ in a sum of two integers: $n_{i}=r_{i}+s_{i}$. If the participants want to commit to bits rather than integers, then $P_{i}$ would split the " 0 " bit as either $0+0$ or $1+1$, and the " 1 " bit as either $0+1$ or $1+0$.

(2) (Commitment phase.) $P_{1}$ sends $r_{1}$ to $P_{2}$, then $P_{2}$ sends $r_{1}+r_{2}$ to $P_{3}$, then $P_{3}$ sends $r_{1}+r_{2}+r_{3}$ to $P_{1}$. In the "opposite direction", $P_{3}$ sends $s_{3}$ to $P_{2}$, then $P_{2}$ sends $s_{2}+s_{3}$ to $P_{1}$, then $P_{1}$ sends $s_{1}+s_{2}+s_{3}$ to $P_{3}$.

After the commitment phase, $P_{1}$ has $s_{1}, s_{2}+s_{3}, r_{1}$, and $r_{1}+r_{2}+r_{3}$ (therefore also $r_{2}+r_{3}$ ), so he cannot possibly recover any $n_{i}$ other than his own. (He can recover $n_{2}+n_{3}$, but this does not give him any information about either $n_{2}$ or $n_{3}$ ). Then, $P_{2}$ has $s_{2}, s_{3}, r_{1}$, and $r_{2}$, so he, too, cannot possibly recover any $n_{i}$ other than his own. Finally, $P_{3}$ has $s_{3}, r_{3}, r_{1}+r_{2}$, and $s_{1}+s_{2}+s_{3}$ (therefore also $s_{1}+s_{2}$ ), so he, too, cannot possibly recover any $n_{i}$ other than his own, (He can recover $n_{1}+n_{2}$, but this does not give him any information about either $n_{1}$ or $n_{2}$ ).

(3) (Decommitment phase starts.) Note that during the decommitment steps below, each participant transmits information that somebody else had committed to before. This way, each piece of transmitted information can be corroborated by two parties, which prevents cheating since we are assuming that no two participants form a coalition.

(4) $P_{3}$ sends $n_{1}+n_{2}$ to both $P_{1}$ and $P_{2}$. Now $P_{1}$ knows $n_{2}$, and $P_{2}$ knows $n_{1}$.

(5) $P_{2}$ sends $r_{1}$ to $P_{3}$. Now $P_{3}$ can recover $r_{2}$ from $r_{1}$ and $r_{1}+r_{2}$.

(6) $P_{1}$ sends $s_{2}+s_{3}$ to $P_{3}$. Now $P_{3}$ can extract $s_{2}$ from this sum, and then, since he has $r_{2}$, recover $n_{2}$, and then also $n_{1}$ since $P_{3}$ already knows $n_{1}+n_{2}$.

(7) $P_{1}$ sends $r_{1}+r_{2}+r_{3}$ to $P_{2}$. Now $P_{2}$ can recover $r_{3}$ and therefore $n_{3}=r_{3}+s_{3}$.

This protocol can be obviously generalized to $3 m$ participants for arbitrary $m \geq 1$ by splitting the players into triples and applying the above protocol to each triple. It can also be generalized to an arbitrary number $k \geq 3$ of participants with a circular arrangement of $k$ secure channels, but we leave details to the reader.

Remark 3. A question that one might now ask, if only out of curiosity, is: would this scheme work with any arrangement of secure channels other than a union of disjoint circuits of length $\geq 3$ ? The answer to this question is "no". Indeed, if in the graph of secure channels there is a vertex (corresponding to $P_{1}$, say) of degree 0 , then any information sent out by $P_{1}$ will be available to everybody, so other participants will know $n_{1}$ unless $P_{1}$ uses a one-way function to conceal it. If there is a vertex (again, corresponding to $P_{1}$ ) of degree 1 , this would mean that $P_{1}$ has a secure channel of communication with just one other participant, say $P_{2}$. Then any information sent out by $P_{1}$ will be available at least to $P_{2}$, so $P_{2}$ will know $n_{1}$ unless $P_{1}$ uses a one-way function to conceal it. So, every vertex in the graph should have degree at least 2 , which 
implies that every vertex is included in a circuit. It follows, in particular, that the total number of secure channels should be at least $k$, by the number of participants.

\section{7. (Bit) COMMitMENT BETWEen tWo PARTiES}

Now we show how our unconditionally secure commitment scheme for 3 parties from Section 6 can be used to arrange an unconditionally secure commitment between just two parties. This is similar, in spirit, to the idea of Rivest [15], where an extra participant is introduced to bring the number of parties up to 3 . However, an important difference between our proposal and that of [15] is that the extra participant in [15] is a "trusted initializer", which means that (i) he is allowed to generate randomness; (ii) he can transmit information to "real" participants over secure channels.

By contrast, our extra participant is a "dummy", i.e., (i) he is not allowed to generate randomness; (ii) he can receive information from "real" participants over secure channels and perform simple arithmetic operations.

One possible real-life interpretation of such a "dummy" would be an online calculator that can combine inputs from different users. Also note that in our scheme below the "dummy" is unaware of the committed values, which is useful in case the two "real" participants do not want their commitments to ever be revealed to the third party; for example, such a "dummy" could be a mediator between two parties negotiating a divorce settlement.

Thus, let A (Alice) and B (Bob) be two "real" participants, and D (Dummy) the "dummy". Suppose A and B want to commit to integers $n_{1}$ and $n_{2}$, respectively.

(1) A and B randomly split their integers $n_{i}$ in a sum of two integers: $n_{i}=r_{i}+s_{i}$.

(2) (Commitment.) A sends $s_{1}$ to B, and B sends $r_{2}$ to A. Then, A sends $r_{1}+r_{2}$ to $\mathrm{D}$, and $\mathrm{B}$ sends $s_{1}+s_{2}$ to $\mathrm{D}$.

(3) (Decommitment.) D reveals $r_{1}+r_{2}+s_{1}+s_{2}=n_{1}+n_{2}$ both to $\mathrm{A}$ and $\mathrm{B}$.

(4) Now A knows $\left(n_{1}+n_{2}\right)-n_{1}=n_{2}$, and B knows $\left(n_{1}+n_{2}\right)-n_{2}=n_{1}$, so cheating by either party is impossible.

\section{8. $k-n$ OBLIVIOUS TRANSFER}

An oblivious transfer protocol is a protocol by which a sender sends some information to the receiver, but remains oblivious as to what is received. The first form of oblivious transfer was introduced in 1981 by Rabin [14]. Rabin's oblivious transfer was later shown to be equivalent to "1-2 oblivious transfer"; the latter was subsequently generalized to $1-n$ oblivious transfer and to $k-n$ oblivious transfer [3]. In the latter case, the receiver obtains a set of $k$ messages from a collection of $n$ messages. The set of $k$ messages may be received simultaneously ("non-adaptively"), or they may be requested consecutively, with each request based on previous messages received. All the aforementioned constructions use encryption, so in particular they use one-way functions. The first proposal that did not use one-way functions (and therefore offered unconditionally secure oblivious transfer) appeared in the paper by Rivest [15] that we have already cited in our Section 7 . 
In this section, we offer an unconditionally secure $k-n$ oblivious transfer protocol that is essentially different from that of Rivest in a similar way that our bit commitment protocol in Section 7 is different from Rivest's unconditionally secure bit commitment protocol [15]. More specifically, the extra participant in [15] is a "trusted initializer", which means, in particular, that (i) he is allowed to generate randomness; (ii) he can "consciously" transmit information to "real" participants over secure channels.

By contrast, our extra participant is a "dummy", i.e., (i) he is not allowed to generate randomness; (ii) he can receive information from "real" participants over secure channels, but he transmits information upon specific requests only.

Again, let A (Alice) and B (Bob) be two "real" participants, and D (Dummy) the "dummy", e.g., a computer. Suppose A has a collection of $n$ messages, and B wants to obtain $k$ of these messages, without A knowing which messages B has received. Suppose that all messages are integers $m_{i}, 1 \leq i \leq n$.

(1) A randomly splits her integers $m_{i}$ in a sum of two integers: $m_{i}=r_{i}+s_{i}$.

(2) A sends the (ordered) set of all $r_{i}, 1 \leq i \leq n$, to $\mathrm{D}$, and the (ordered) set of all $s_{i}, 1 \leq i \leq n$, to $\mathrm{B}$.

(3) B sends to $\mathrm{D}$ the set of indices $j_{1}, \ldots, j_{k}$ corresponding to the messages $m_{j}$ he wants to receive.

(4) $\mathrm{D}$ sends to B the (ordered) set $r_{j_{1}}, \ldots, r_{j_{k}}$.

(5) B recovers $m_{j_{1}}, \ldots, m_{j_{k}}$ as a sum of relevant $r_{j}$ and $s_{j}$.

\section{Mental poker}

"Mental poker" is the common name for a set of cryptographic problems that concerns playing a fair game over distance without the need for a trusted third party. One of the ways to describe the problem is: how can 2 players deal cards fairly over the phone? Several protocols for doing this have been suggested, including [17], [5], [9] and [1]. As with the bit commitment, it is rather obvious that a fair card dealing to two players over distance is impossible without a one-way function, or even a one-way function with trapdoor. However, it turns out to be possible if the number of players is at least 3, assuming, of course, that there are secure channels for communication between at least some of the players. In our proposal, we will be using $k$ secure channels for $k \geq 3$ players $P_{1}, \ldots, P_{k}$, and these $k$ channels will be arranged in a circuit: $P_{1} \rightarrow P_{2} \rightarrow \ldots \rightarrow P_{k} \rightarrow P_{1}$.

To begin with, suppose there are 3 players: $P_{1}, P_{2}$, and $P_{3}$ and 3 secure channels: $P_{1} \rightarrow P_{2} \rightarrow P_{3} \rightarrow P_{1}$.

The first protocol, Protocol 1 below, is for distributing all integers from 1 to $m$ to the players in such a way that each player gets about the same number of integers. (For example, if the deck that we want to deal has 52 cards, then two players should get 17 integers each, and one player should get 18 integers.) In other words, Protocol 1 allows one to randomly split a set of $m$ integers into 3 disjoint sets.

The second protocol, Protocol 2, is for collectively generating random integers modulo a given integer $M$. This very simple but useful primitive can be used: (i) for collectively generating, uniformly randomly, a permutation from the group $S_{m}$. This 
will allow us to assign cards from a deck of $m$ cards to the $m$ integers distributed by Protocol 1; (ii) introducing "dummy" players as well as for "playing" after dealing cards.

9.1. Protocol 1. For notational convenience, we are assuming below that we have to distribute integers from 1 to $r=3 s$ to 3 players.

To begin with, all players agree on a parameter $N$, which is a positive integer of a reasonable magnitude, say, 10.

(1) each player $P_{i}$ picks, uniformly randomly, an integer (a "counter") $c_{i}$ between 1 and $N$, and keeps it private.

(2) $P_{1}$ starts with the "extra" integer 0 and sends it to $P_{2}$.

(3) $P_{2}$ sends to $P_{3}$ either the integer $m$ he got from $P_{1}$, or $m+1$. More specifically, if $P_{2}$ gets from $P_{1}$ the same integer $m$ less than or equal to $c_{2}$ times, then he sends $m$ to $P_{3}$; otherwise, he sends $m+1$ and keeps $m$ (i.e., in the latter case $m$ becomes one of "his" integers). Having sent out $m+1$, he "resets his counter", i.e., selects, uniformly randomly between 1 and $N$, a new $c_{2}$. He also resets his counter if he gets the number $m$ for the first time, even if he does not keep it.

(4) $P_{3}$ sends to $P_{1}$ either the integer $m$ he got from $P_{2}$, or $m+1$. More specifically, if $P_{3}$ gets from $P_{2}$ the same integer $m$ less than or equal to $c_{3}$ times, then he sends $m$ to $P_{1}$; otherwise, he sends $m+1$ and keeps $m$. Having sent out $m+1$, he selects a new counter $c_{3}$. He also resets his counter if he gets the number $m$ for the first time, even if he does not keep it.

(5) $P_{1}$ sends to $P_{2}$ either the integer $m$ he got from $P_{3}$, or $m+1$. More specifically, if $P_{1}$ gets from $P_{3}$ the same integer $m$ less than or equal to $c_{1}$ times, then he sends $m$ to $P_{2}$; otherwise, he sends $m+1$ and keeps $m$. Having sent out $m+1$, he selects a new counter $c_{1}$. He also resets his counter if he gets the number $m$ for the first time, even if he does not keep it.

(6) This procedure continues until one of the players gets $s$ integers (not counting the "extra" integer 0). After that, a player who already has $s$ integers just "passes along" any integer that comes his way, while other players keep following the above procedure until they, too, get $s$ integers.

(7) The protocol ends as follows. When all $3 s$ integers, between 1 and $3 s$, are distributed, the player who got the last integer, $3 \mathrm{~s}$, keeps this fact to himself and passes this integer along as if he did not "take" it.

(8) The process ends when the integer $3 s$ makes $N+1$ "full circles".

We note that the role of the "extra" integer 0 is to prevent $P_{3}$ from knowing that $P_{2}$ has got the integer 1 if it happens so that $c_{2}=1$ in the beginning.

We also note that this protocol can be generalized to arbitrarily many players in the obvious way, if there are $k$ secure channels for communication between $k$ players, arranged in a circuit.

9.2. Protocol 2. Now we describe a protocol for generating random integers modulo some integer $M$ collectively by 3 players. As in Protocol 1, we are assuming that there are secure channels for communication between the players, arranged in a circuit. 
(1) $P_{2}$ and $P_{3}$ uniformly randomly and independently select private integers $n_{2}$ and $n_{3}$ (respectively) modulo $M$.

(2) $P_{2}$ sends $n_{2}$ to $P_{1}$, and $P_{3}$ sends $n_{3}$ to $P_{1}$.

(3) $P_{1}$ computes the sum $m=n_{2}+n_{3}$ modulo $M$.

Note that neither $P_{2}$ nor $P_{3}$ can cheat by trying to make a "clever" selection of their $n_{i}$ because the sum, modulo $M$, of any integer with an integer uniformly distributed between 0 and $M-1$, is an integer uniformly distributed between 0 and $M-1$.

Finally, $P_{1}$ cannot cheat simply because he does not really get a chance: if he miscalculates $n_{2}+n_{3}$ modulo $M$, this will be revealed at the end of the game. (All players keep contemporaneous records of all transactions, so that at the end of the game, correctness could be verified.)

To generalize Protocol 2 to arbitrarily many players $P_{1}, \ldots, P_{k}, k \geq 3$, we can just engage 3 players at a time in running the above protocol. If, at the same time, we want to keep the same circular arrangement of secure channels between the players that we had in Protocol 1, i.e., $P_{1} \rightarrow P_{2} \rightarrow \ldots P_{k} \rightarrow P_{1}$, then 3 players would have to be $P_{i+1}$, $P_{i}, P_{i+2}$, where $i$ would run from 1 to $k$, and the indices are considered modulo $k$.

Protocol 2 can now be used to collectively generate, uniformly randomly, a permutation from the group $S_{m}$. This will allow us to assign cards from a deck of $m$ cards to the $m$ integers distributed by Protocol 1. Generating a random permutation from $S_{m}$ can be done by taking a random integer between 1 and $m$ (using Protocol 2) sequentially, ensuring that there is no repetition. This "brute-force" method will require occasional retries whenever the random integer picked is a repeat of an integer already selected. A simple algorithm to generate a permutation from $S_{m}$ uniformly randomly without retries, known as the Knuth shuffle, is to start with the identity permutation or any other permutation, and then go through the positions 1 through $(m-1)$, and for each position $i$ swap the element currently there with an arbitrarily chosen element from positions $i$ through $m$, inclusive (again, Protocol 2 can be used here to produce a random integer between $i$ and $m$ ). It is easy to verify that any permutation of $m$ elements will be produced by this algorithm with probability exactly $\frac{1}{m !}$, thus yielding a uniform distribution over all such permutations.

After this is done, we have $m$ cards distributed uniformly randomly to the players, i.e., we have:

Proposition 1. If $m$ cards are distributed to $k$ players using Protocols 1 and 2, then the probability for any particular card to be distributed to any particular player is $\frac{1}{k}$.

9.3. Using "dummy" players while dealing cards. We now show how a combination of Protocol 1 and Protocol 2 can be used to deal cards to just 2 players. If we have 2 players, they can use a "dummy" player (e.g. a computer), deal cards to 3 players as in Protocol 1, and then just ignore the "dummy"'s cards, i.e., "put his cards back in the deck". We note that the "dummy" in this scenario would not generate randomness; it will be generated for him by the other two players using Protocol 2. Namely, if we call the "dummy" $P_{3}$, then the player $P_{1}$ would randomly generate $c_{31}$ between 1 and $N$ and send it to $P_{3}$, and $P_{2}$ would randomly generate $c_{32}$ between 1 and $N$ and send it to $P_{3}$. Then $P_{3}$ would compute his random number as $c_{3}=c_{31}+c_{32}$ modulo $N$. 
Similarly, "dummy" players can help $k$ "real" players each get a fixed number $s$ of cards, because Protocol 1 alone is only good for distributing all cards in the deck to the players, dealing each player about the same number of cards. We can introduce $m$ "dummy" players so that $(m+k) \cdot s$ is approximately equal to the number of cards in the deck, and position all the "dummy" players one after another as part of a circuit $P_{1} \rightarrow P_{2} \rightarrow \ldots P_{m+k} \rightarrow P_{1}$. Then we use Protocol 1 to distribute all cards in the deck to $(m+k)$ players taking care that each "real" player gets exactly $s$ cards. As in the previous paragraph, "dummy" players have "real" ones generate randomness for them using Protocol 2.

After all cards in the deck are distributed to $(m+k)$ players, "dummy" players send all their cards to one of them; this "dummy" player now becomes a "dummy dealer", i.e., he will give out random cards from the deck to "real" players as needed in the course of a subsequent game, while randomness itself will be supplied to him by "real" players using Protocol 2.

10. Summary of the properties of our card Dealing (Protocols 1 and 2)

Here we summarize the properties of our Protocols 1 and 2 and compare, where appropriate, our protocols to the card dealing protocol of [1].

1. Uniqueness of cards. Yes, by the very design of Protocol 1.

2. Uniform random distribution of cards. Yes, because of Protocol 2; see our Proposition 1 in Section 9.2.

3. Complete confidentiality of cards. Yes, by the design of Protocol 1.

4. Number of secure channels for communication between $k \geq 3$ players: $k$, arranged in a circuit.

By comparison, the card dealing protocol of [1] requires $3 k$ secure channels.

5. Average number of transmissions between $k \geq 3$ players: $O\left(\frac{N}{2} m k\right)$, where $m$ is the number of cards in the deck, and $N \approx 10$. This is because in Protocol 1 , the number of circles (complete or incomplete) each integer makes is either 1 or the minimum of all the counters $c_{i}$ at the moment when this integer completes the first circle. Since the average of $c_{i}$ is at most $\frac{N}{2}$, we get the result because within one circle (complete or incomplete) there are at most $k$ transmissions. We note that in fact, there is a precise formula for the average of the minimum of $c_{i}$ in this situation: $\frac{\sum_{j=1}^{N} j^{k}}{N^{k}}$, which is less than $\frac{N}{2}$ if $k \geq 2$.

By comparison, in the protocol of [1] there are $O\left(m k^{2}\right)$ transmissions.

6. Total length of transmissions between $k \geq 3$ players: $\frac{N}{2} m k \cdot \log _{2} m$ bits. This is just the average number of transmissions times the length of a single transmission, which is a positive integer between 1 and $m$.

By comparison, total length of transmissions in [1] is $O\left(m k^{2} \log k\right)$.

7. Computational cost of Protocol 1: 0 (because there are no computations, only transmissions). 
By comparison, the protocol of [1] requires computing products of up to $k$ permutations from the group $S_{k}$ to deal just one card; the total computational cost therefore is $O\left(m k^{2} \log k\right)$.

\section{SeCret SHARING}

Secret sharing refers to method for distributing a secret amongst a group of participants, each of whom is allocated a share of the secret. The secret can be reconstructed only when a sufficient number of shares are combined together; individual shares are of no use on their own.

More formally, in a secret sharing scheme there is one dealer and $k$ players. The dealer gives a secret to the players, but only when specific conditions are fulfilled. The dealer accomplishes this by giving each player a share in such a way that any group of $t$ (for threshold) or more players can together reconstruct the secret but no group of fewer than $t$ players can. Such a system is called a $(t, k)$-threshold scheme (sometimes written as a $(k, t)$-threshold scheme).

Secret sharing was invented by Shamir [16] and Blakley [2], independent of each other, in 1979. Both proposals assumed secure channels for communication between the dealer and each player. In our proposal here, the number of secure channels is equal to $2 k$, where $k$ is the number of players, because in addition to the secure channels between the dealer and each player, we have $k$ secure channels for communication between the players, arranged in a circuit: $P_{1} \rightarrow P_{2} \rightarrow \ldots \rightarrow P_{k} \rightarrow P_{1}$.

The advantage over Shamir's and other known secret sharing schemes that we are going to get here is that nobody, including the dealer, ends up knowing the shares (of the secret) owned by any particular players. The disadvantage is that our scheme is a $(k, k)$-threshold scheme only.

We start by describing a subroutine for distributing shares by the players among themselves. More precisely, $k$ players want to split a given number in a sum of $k$ numbers, so that each summand is known to one player only, and each player knows one summand only.

11.1. The Subroutine (distributing shares by the players among themselves). Suppose a player $P_{i}$ receives a number $M$ that has to be split in a sum of $k$ private numbers. In what follows, all indices are considered modulo $k$.

(1) $P_{i}$ initiates the process by sending $M-m_{i}$ to $P_{i+1}$, where $m_{i}$ is a random number (could be positive or negative).

(2) Each subsequent $P_{j}$ does the following. Upon receiving a number $m$ from $P_{j-1}$, he subtracts a random number $m_{j}$ from $m$ and sends the result to $P_{j+1}$. The number $m_{j}$ is now $P_{j}$ 's secret summand.

(3) When this process gets back to $P_{i}$, he adds $m_{i}$ to whatever he got from $P_{i-1}$; the result is his secret summand.

Now we get to the actual secret sharing protocol. 
11.2. The protocol (secret sharing $(k, k)$-threshold scheme). The dealer $D$ wants to distribute shares of a secret number $N$ to $k$ players $P_{i}$ so that, if $P_{i}$ gets a number $s_{i}$, then $\sum_{i=1}^{k} s_{i}=N$.

(1) $D$ arbitrarily splits $N$ in a sum of $k$ integers: $N=\sum_{i=1}^{k} n_{i}$.

(2) The loop: at Step $i$ of the loop, $D$ sends $n_{i}$ to $P_{i}$, and $P_{i}$ initiates the above Subroutine to distribute shares $n_{i j}$ of $n_{i}$ among the players, so that $\sum_{j=1}^{k} n_{i j}=$ $n_{i}$.

(3) After all $k$ steps of the loop are completed, each player $P_{i}$ ends up with $k$ numbers $n_{j i}$ that sum up to $s_{i}=\sum_{j=1}^{k} n_{j i}$. It is obvious that $\sum_{i=1}^{k} s_{i}=N$.

Acknowledgement. Both authors are grateful to Max Planck Institut für Mathematik, Bonn for its hospitality during the work on this paper.

\section{REFERENCES}

[1] I. Bárány, Z. Füredi, Mental poker with three or more players, Inform. and Control 59 (1983), 84-93.

[2] G. R. Blakley, Safeguarding cryptographic keys, Proceedings of the National Computer Conference 48 (1979), 313-317.

[3] G. Brassard, C. Crépeau and J.-M. Robert, All-or-nothing disclosure of secrets, In Advances in Cryptology - CRYPTO '86, pp. 234-238, Lecture Notes Comp. Sc. 263, Springer, 1986.

[4] D. Chaum, C. Crépeau, and I. Damgard, Multiparty unconditionally secure protocols (extended abstract), Proceedings of the Twentieth ACM Symposium on the Theory of Computing, ACM, 1988, pp. 11-19.

[5] C. Crépeau, A zero-knowledge poker protocol that achieves confidentiality of the players' strategy or how to achieve an electronic poker face, Advances in cryptology - CRYPTO '86, pp. 239-247, Lecture Notes Comp. Sc. 263, Springer, 1986.

[6] I. Damgard, M. Geisler, M. Kroigard, Homomorphic encryption and secure comparison, Int. J. Appl. Cryptogr. 1 (2008), 22-31.

[7] I. Damgard, Y. Ishai, Scalable secure multiparty computation, Advances in cryptology CRYPTO 2006, 501-520, Lecture Notes in Comput. Sci. 4117, Springer, Berlin, 2006.

[8] O. Goldreich, Foundations of Cryptography: Volume 1, Basic Tools. Cambridge University Press, 2007.

[9] S. Goldwasser and S. Micali, Probabilistic Encryption and How to Play Mental Poker Keeping Secret All Partial Information, in Proceedings of the 14th Annual ACM symp. on Theory of computing, ACM-SIGACT, May 1982, pp. 365-377.

[10] S. Goldwasser, S. Micali, Probabilistic encryption, J. Comput. System Sci. 28 (1984), 270-299.

[11] D. Grigoriev, I. Ponomarenko, Constructions in public-key cryptography over matrix groups, Contemp. Math., Amer. Math. Soc. 418 (2006), 103-119.

[12] R. Impagliazzo and M. Luby, One-way functions are essential for complexity based cryptography, in: FOCS'89, IEEE Computer Society, 1989, pp. 230-235.

[13] A. Menezes, P. van Oorschot, and S. Vanstone, Handbook of Applied Cryptography, CRC-Press 1996.

[14] M. Rabin, How to exchange secrets by oblivious transfer, Technical Report TR-81, Aiken Computation Laboratory, Harvard University, 1981.

[15] R. Rivest, Unconditionally Secure Commitment and Oblivious Transfer Schemes Using Private Channels and a Trusted Initializer, preprint, 1999.

[16] A. Shamir, How to share a secret, Comm. ACM 22 (1979), 612-613. 
[17] A. Shamir, R. Rivest, and L. Adleman, Mental poker, Technical Report LCS/TR-125, Massachusetts Institute of Technology, April 1979.

[18] A. C. Yao, Protocols for secure computations (Extended Abstract), 23rd annual symposium on foundations of computer science (Chicago, Ill., 1982), 160-164, IEEE, New York, 1982.

CNRS, Mathématiques, Université de Lille, 59655, Villeneuve D'AscQ, France

E-mail address: dmitry.grigoryev@math.univ-lille1.fr

Department of Mathematics, The City College of New York, New York, Ny 10031

E-mail address: shpil@groups.sci.ccny.cuny.edu 